Title : will be set by the publisher

Editors : will be set by the publisher

EAS Publications Series, Vol. ?, 2018

\title{
ANALYZING DIRECT DARK MATTER DETECTION DATA BY THE AMIDAS WEBSITE
}

\author{
Chung-Lin Shan ${ }^{1}$
}

\begin{abstract}
In this talk I have presented the data analysis results of extracting properties of halo WIMPs: the mass and the (ratios between the) spin-independent and spin-dependent couplings/cross sections on nucleons by the AMIDAS website. Although non-standard astronomical setup has been used to generate pseudodata sets for our analyses, it has been found that, without prior information/assumption about the local density and velocity distribution of halo Dark Matter, these WIMP properties have been reconstructed with $\sim 5 \%$ to $\lesssim 40 \%$ deviations from the input values.
\end{abstract}

\section{Introduction}

In order to extract properties of halo WIMPs (Weakly Interacting Massive Particles) by using data from direct Dark Matter detection experiments as modelindependently as possible, we have developed a series of data analysis method for reconstructing the one-dimensional WIMP velocity distribution function (Drees \& Shan 2007) as well as determining the WIMP mass (Drees \& Shan 2008), the spin-independent (SI) WIMP coupling on nucleons (Shan 2011) and the ratios between different WIMP couplings/cross sections (Shan 2011). Moreover, in collaboration with the DAMNED (DArk Matter Network Exclusion Diagram) Dark Matter online tool (DAMNED), part of the ILIAS Project (ILIAS), the "AMIDAS" (A Model-Independent Data Analysis System) website for online simulation/data analysis has also been established (AMIDAS; Shan 2010, 2009).

In this article, in order to demonstrate the usefulness and powerfulness as well as the model-independence of the AMIDAS package for direct Dark Matter detection experiments, I will analyze blindly some pseudodata sets generated for different detector materials and present the reconstructed WIMP properties. This

\footnotetext{
1 Institute of Physics, Academia Sinica, No. 128, Sec. 2, Academia Road, Nankang, Taipei 11529, Taiwan, R.O.C.

E-mail: clshan@phys.sinica.edu.tw
} 
Title : will be set by the publisher

means that I will simply upload these data sets onto the AMIDAS website and follow the instructions to reconstruct different WIMP properties without using any information about the input setup used for generating the pseudodata. For cases in which some information about WIMPs (e.g., the mass $m_{\chi}$ ) and/or Galactic halo (e.g., the local Dark Matter density $\rho_{0}$ ) is required, I will naively use the commonly used/favorite values for the data analyses.

After that I show the blindly reconstructed properties of halo WIMPs in Sec. 2, in Sec. 3 I will reveal the input setup used for generating the analyzed data and compare the reconstructed results to them. Finally, I conclude in Sec. 4.

\section{Reconstructed WIMP properties}

In this section, I present the reconstructed WIMP properties analyzed by the AMIDAS website. While in each uploaded file there are exactly 50 data sets, in each data set there are on average 50 recorded events (i.e., 50 measured recoil energies $)^{1}$; the exact number of total events is Poisson distributed. For simplicity, the experimental minimal and maximal cut-off energies have been set as 0 and $100 \mathrm{keV}$ for all data sets.

In order to check the effect of using a "wrong" elastic nuclear form factor, two forms have been considered for the SI WIMP-nucleus cross section in our analyses. One is the simple exponential form:

$$
F_{\mathrm{ex}}^{2}(Q)=e^{-Q / Q_{0}}
$$

Here $Q$ is the recoil energy transferred from the incident WIMP to the target nucleus, $Q_{0}$ is the nuclear coherence energy given by $Q_{0}=1.5 / m_{\mathrm{N}} R_{0}^{2}$, where $R_{0}=\left[0.3+0.91\left(m_{\mathrm{N}} / \mathrm{GeV}\right)^{1 / 3}\right] \mathrm{fm}$ is the radius of the nucleus and $m_{\mathrm{N}}$ is the mass of the target nucleus. Meanwhile, we used also a more realistic analytic form for the elastic nuclear form factor:

$$
F_{\mathrm{SI}}^{2}(Q)=\left[\frac{3 j_{1}\left(q R_{1}\right)}{q R_{1}}\right]^{2} e^{-(q s)^{2}}
$$

Here $j_{1}(x)$ is a spherical Bessel function, $q=\sqrt{2 m_{\mathrm{N}} Q}$ is the transferred 3momentum, for the effective nuclear radius we use $R_{1}=\sqrt{R_{A}^{2}-5 s^{2}}$ with $R_{A} \simeq$ $1.2 A^{1 / 3} \mathrm{fm}$ and a nuclear skin thickness $s \simeq 1 \mathrm{fm}$. For the SD WIMP-nucleus cross section, we only used the "thin-shell" nuclear form factor:

$$
F_{\mathrm{TS}}^{2}(Q)= \begin{cases}j_{0}^{2}\left(q R_{1}\right), & \text { for } q R_{1} \leq 2.55 \text { or } q R_{1} \geq 4.5 \\ \text { const. } \simeq 0.047, & \text { for } 2.55 \leq q R_{1} \leq 4.5\end{cases}
$$



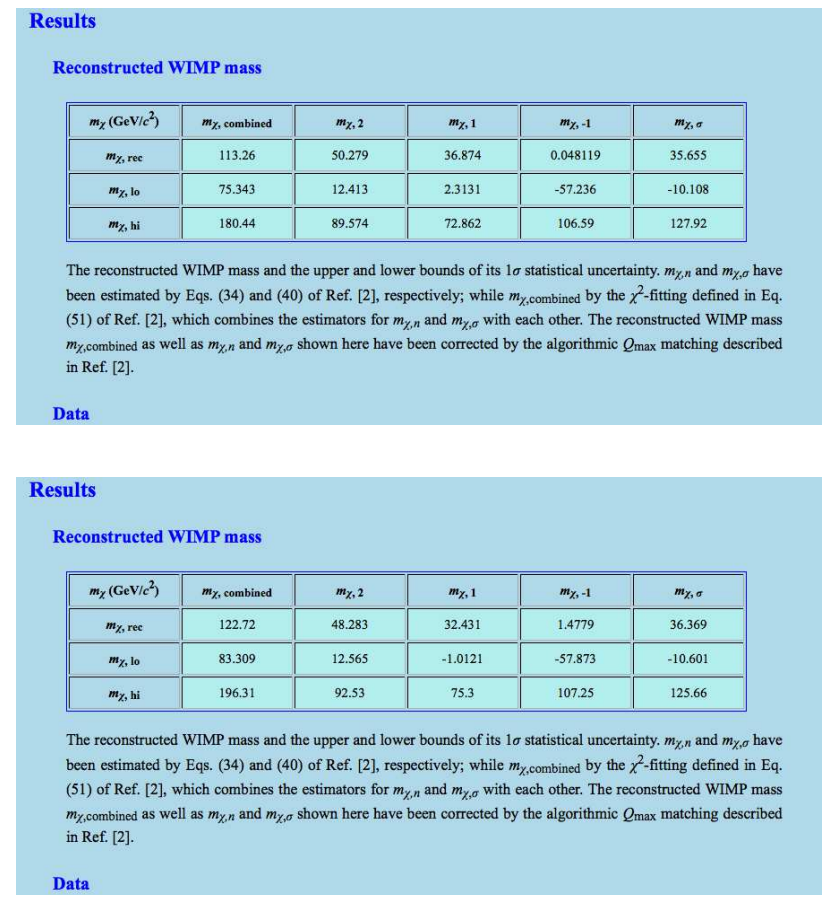

Fig. 1. The WIMP mass $m_{\chi}$ reconstructed with a target combination of ${ }^{28} \mathrm{Si}+{ }^{76} \mathrm{Ge}$ nuclei. Two forms of the elastic nuclear form factor given in Eqs. (2.1) and (2.2) have been used in the upper and lower frames, respectively.

\subsection{WIMP mass $m_{\chi}$}

As one of the most important properties of halo WIMPs as well as the basic information for reconstructing other quantities in our model-independent analysis methods, I consider at first the determination of the WIMP mass $m_{\chi}$ by means of the method introduced in Drees \& Shan (2008).

In Figs. $1 \mathrm{I}$ show the reconstructed WIMP masses and the upper and lower bounds of their $1 \sigma$ statistical uncertainties. The usual target combination of ${ }^{28} \mathrm{Si}+{ }^{76} \mathrm{Ge}$ nuclei has been used for this reconstruction, whereas two forms of the elastic nuclear form factor given in Eqs. (2.1) and (2.2) have been used for determining $m_{\chi}$ in the upper and lower frames, respectively. While $m_{\chi, n}$ with $n=-1,1,2$ and $m_{\chi, \sigma}$ have been estimated by Eqs. (34) and (40) of Drees \& Shan (2008), respectively, $m_{\chi, \text { combined }}$ has been estimated by the $\chi^{2}$-fitting defined in Eq. (51) of Drees \& Shan (2008), which combines the estimators for $m_{\chi, n}$ and $m_{\chi, \sigma}$ with each other. The reconstructed WIMP mass $m_{\chi, \text { combined }}$ as well as $m_{\chi, n}$ and $m_{\chi, \sigma}$ shown here have been corrected by the iterative $Q_{\max }$-matching procedure

\footnotetext{
${ }^{1}$ Note that we considered here only data sets with pure WIMP signals, possible unrejected background events are neglected.
} 
Title : will be set by the publisher

described in Drees \& Shan (2008).

It can be found here that, although all single estimators $\left(m_{\chi, n}\right.$ with $n=-1,1$, 2 and $m_{\chi, \sigma}$ ) give generally a (relatively lighter) WIMP mass of $\sim 50 \mathrm{GeV}$ or even lighter and a $1 \sigma$ upper bound of $\sim 130 \mathrm{GeV}$, the mean values of the combined (in principle, more reliable) results (the second column in two tables) of the reconstructed WIMP mass give $m_{\chi} \sim 120 \mathrm{GeV}$ with a rough $1 \sigma$ upper (lower) bound of $\sim 190$ (80) GeV, or, equivalently,

$$
m_{\chi} \simeq 120_{-40}^{+70} \mathrm{GeV} .
$$

Moreover, the combined results with two different form factors show not only a large overlap between $\sim 85 \mathrm{GeV}$ and $\sim 180 \mathrm{GeV}$, but also a good coincidence: comparing to the $\sim_{-40}^{+70} \mathrm{GeV} 1 \sigma$ statistical uncertainty and the $\sim_{-35}^{+60} \mathrm{GeV}$ overlap, the difference between two median values is $\lesssim 10 \mathrm{GeV}$ ! This indicates that, for the first approximation of giving/constraining the most plausible range of the WIMP mass, the uncertainty on the nuclear form factor could be safely neglected.

\subsection{Spin-independent WIMP-nucleon coupling $\left|f_{\mathrm{p}}\right|^{2}$}

Following the WIMP mass determination, I consider now the reconstruction of the SI WIMP coupling on nucleons $\left|f_{\mathrm{p}}\right|^{2}$ (Shan 2011) with a ${ }^{76}$ Ge target ${ }^{2}$.

In Figs. 2 I show the reconstructed squared SI WIMP-nucleon couplings and the lower and upper bounds of their $1 \sigma$ statistical uncertainties estimated by Eqs. (17) and (18) of Shan (2011) with an assumed (100 $\pm 10 \mathrm{GeV}$, labeled with the subscript "input") and the reconstructed (from Sec. 2.1, labeled with "recon") WIMP masses. The commonly used value of the local Dark Matter density $\rho_{0}=0.3 \mathrm{GeV} / \mathrm{cm}^{3}$ and a larger value of $\rho_{0}=0.4 \mathrm{GeV} / \mathrm{cm}^{3}$ (Catena \& Ullio 2010; Salucci et al. 2010; Pato et al. 2010) as well as the elastic nuclear form factors given in Eqs. (2.1) and (2.2) have been used for estimating $\left|f_{\mathrm{p}}\right|^{2}$ in the upper and lower frames, respectively.

Among these results, the mean value and the overlap of two most plausible results (estimated by using the reconstructed WIMP mass) give roughly (and somehow naively) a $1 \sigma$ range of

$$
\left|f_{\mathrm{p}}\right|^{2} \simeq 9.00_{-1.44}^{+2.10} \times 10^{-18} \mathrm{GeV}^{-4}
$$

or, equivalently,

$$
\left|f_{\mathrm{p}}\right| \simeq 3.00_{-0.24}^{+0.35} \times 10^{-9} \mathrm{GeV}^{-2} .
$$

Since the reconstructed WIMP mass given in Sec. 2.1 is $m_{\chi} \sim 120 \mathrm{GeV}$, one can simply use the proton mass $m_{\mathrm{p}}$ to approximate the WIMP-proton reduced mass

\footnotetext{
${ }^{2}$ Remind that the theoretical prediction by most supersymmetric models that the SI scaler WIMP couplings on protons and on neutrons are (approximately) equal: $f_{\mathrm{p}} \simeq f_{\mathrm{n}}$ has been adopted in the AMIDAS package.
} 

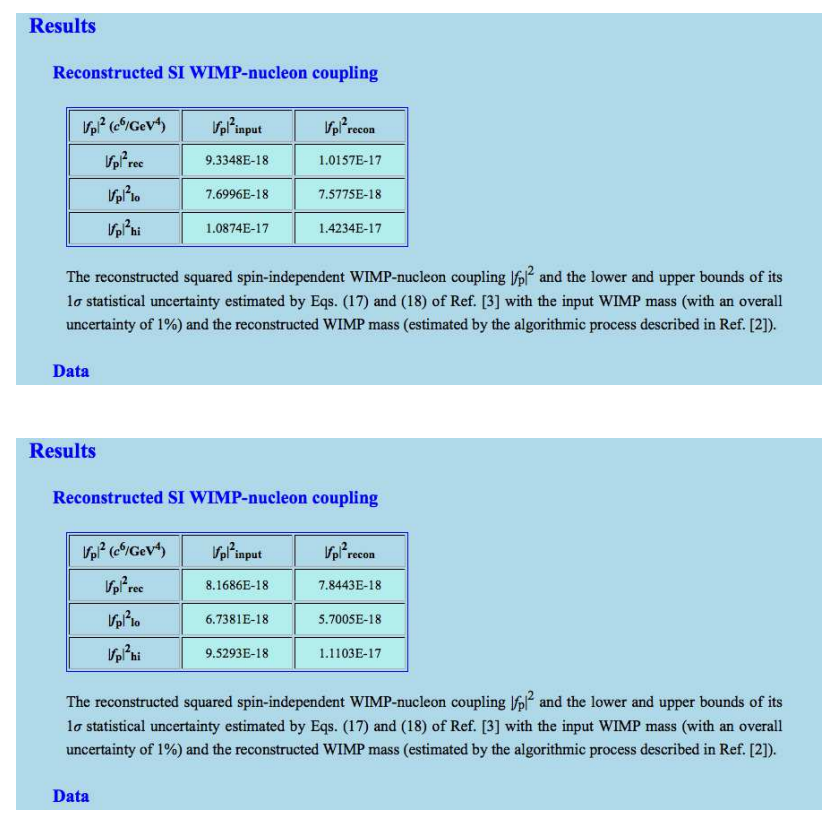

Fig. 2. The squared SI WIMP-nucleon coupling $\left|f_{\mathrm{p}}\right|^{2}$ reconstructed with a ${ }^{76} \mathrm{Ge}$ target. The commonly used value of the local Dark Matter density $\rho_{0}=0.3 \mathrm{GeV} / \mathrm{cm}^{3}$ and a larger value of $\rho_{0}=0.4 \mathrm{GeV} / \mathrm{cm}^{3}$ as well as the elastic nuclear form factors given in Eqs. (2.1) and (2.2) have been used for estimating $\left|f_{\mathrm{p}}\right|^{2}$ in the upper and lower frames, respectively.

$m_{\mathrm{r}, \mathrm{p}}$ and give a reconstructed SI WIMP-nucleon cross section $\mathrm{as}^{3}$

$$
\sigma_{\chi \mathrm{p}}^{\mathrm{SI}}=\left(\frac{4}{\pi}\right) m_{\mathrm{r}, \mathrm{p}}^{2}\left|f_{\mathrm{p}}\right|^{2} \approx\left(\frac{4}{\pi}\right) m_{\mathrm{p}}^{2}\left|f_{\mathrm{p}}\right|^{2} \simeq 4.31_{-0.69}^{+1.01} \times 10^{-9} \mathrm{pb} .
$$

${ }^{3}$ Note that, since the expression for estimating $\left|f_{\mathrm{p}}\right|^{2}$ (Eq. (17) of Shan (2011)) is a function of the (reconstructed) WIMP mass, for light WIMP mass, one has to use

$$
\sigma_{\chi \mathrm{p}}^{\mathrm{SI}}=\frac{1}{\rho_{0}}\left[\frac{1}{\sqrt{2}}\left(\frac{1}{\mathcal{E} A^{2} \sqrt{m_{\mathrm{N}}}}\right)\right]\left[\frac{2 Q_{\min }^{1 / 2} r\left(Q_{\min }\right)}{F^{2}\left(Q_{\min }\right)}+I_{0}\right]\left(m_{\chi}+m_{\mathrm{N}}\right)\left(\frac{m_{\chi} m_{\mathrm{p}}}{m_{\chi}+m_{\mathrm{p}}}\right)^{2},
$$

where $A$ is the atomic mass number of the target nucleus, $\mathcal{E}$ is the experimental exposure. Then one has (cf. Eq. (18) of Shan (2011))

$$
\begin{aligned}
\sigma\left(\sigma_{\chi \mathrm{P}}^{\mathrm{SI}}\right)=\sigma_{\chi \mathrm{P}}^{\mathrm{SI}}\left\{\frac{\sigma^{2}\left(m_{\chi}\right)}{\left(m_{\chi}+m_{\mathrm{N}}\right)^{2}}\right. & {\left[1+\Delta\left(m_{\chi}\right)\right]^{2}+\mathcal{N}_{\mathrm{m}}^{2} \sigma^{2}\left(1 / \mathcal{N}_{\mathrm{m}}\right) } \\
+ & \left.\frac{2 \mathcal{N}_{\mathrm{m}} \operatorname{cov}\left(m_{\chi}, 1 / \mathcal{N}_{\mathrm{m}}\right)}{\left(m_{\chi}+m_{\mathrm{N}}\right)}\left[1+\Delta\left(m_{\chi}\right)\right]\right\}^{1 / 2} .
\end{aligned}
$$

Here I have used (Drees \& Shan 2007)

and defined

$$
\mathcal{N}_{\mathrm{m}}=\left[\frac{2 Q_{\min }^{1 / 2} r\left(Q_{\min }\right)}{F^{2}\left(Q_{\min }\right)}+I_{0}\right]^{-1}
$$

$$
\Delta\left(m_{\chi}\right)=2\left(\frac{m_{\mathrm{p}}}{m_{\chi}}\right)\left(\frac{m_{\chi}+m_{\mathrm{N}}}{m_{\chi}+m_{\mathrm{p}}}\right) \text {. }
$$


Title : will be set by the publisher
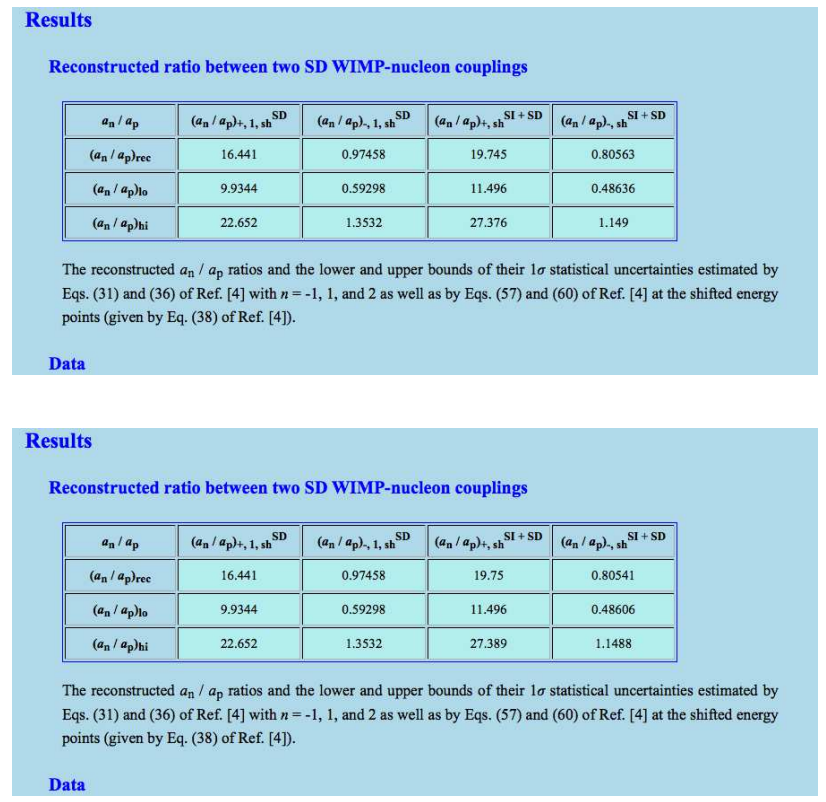

Fig. 3. The reconstructed ratio between two SD WIMP-nucleon couplings, $a_{\mathrm{n}} / a_{\mathrm{p}}$. As usual, the elastic nuclear form factors given in Eqs. (2.1) and (2.2) have been used for determining $a_{\mathrm{n}} / a_{\mathrm{p}}$ in the upper and lower frames, respectively.

\subsection{Ratio of two spin-dependent WIMP-nucleon couplings $a_{\mathrm{n}} / a_{\mathrm{p}}$}

In Figs. 3 I show the reconstructed $a_{\mathrm{n}} / a_{\mathrm{p}}$ ratios and the lower and upper bounds of their $1 \sigma$ statistical uncertainties estimated by Eqs. (2.7) and (2.12) of Shan (2011) with $n=1$ as well as by Eqs. (3.16) and (3.20) of Shan (2011) at the shifted energy points (Drees \& Shan 2007; Shan 2011). A combination of ${ }^{19} \mathrm{~F}+{ }^{127} \mathrm{I}$ targets has been used for the reconstruction of $a_{\mathrm{n}} / a_{\mathrm{p}}$ under the assumption that the SD WIMP-nucleus interaction dominates over the SI one (labeled with the superscript "SD"), whereas a third target of ${ }^{28} \mathrm{Si}$ has been combined with ${ }^{19} \mathrm{~F}$ and ${ }^{127}$ I for the case of the general combination of both SI and SD WIMP interactions (labeled with the superscript "SI + SD").

It can be found that, firstly, the "+ (plus)" solutions of the $a_{\mathrm{n}} / a_{\mathrm{p}}$ ratios given here are obviously too large to be the reasonable choice for $a_{\mathrm{n}} / a_{\mathrm{p}}$ and the "- (minus)" solutions should be the correct ones ${ }^{4}$. Secondly, although the reconstructed result under the assumption of the SD dominant WIMP interaction is in general larger than the (in principle more plausible) result obtained without such a prior

\footnotetext{
Definitions and estimations of $r\left(Q_{\min }\right)$ and $I_{n}$ can be found in e.g., Drees \& Shan $(2007,2008)$.

${ }^{4}$ Remind that, as discussed in Shan (2011), the correct choice from the "+" and "-" solutions can be decided directly by the values of the group spins of protons and neutrons of the used target nuclei, $\left\langle S_{(\mathrm{p}, \mathrm{n})}\right\rangle$.
} 

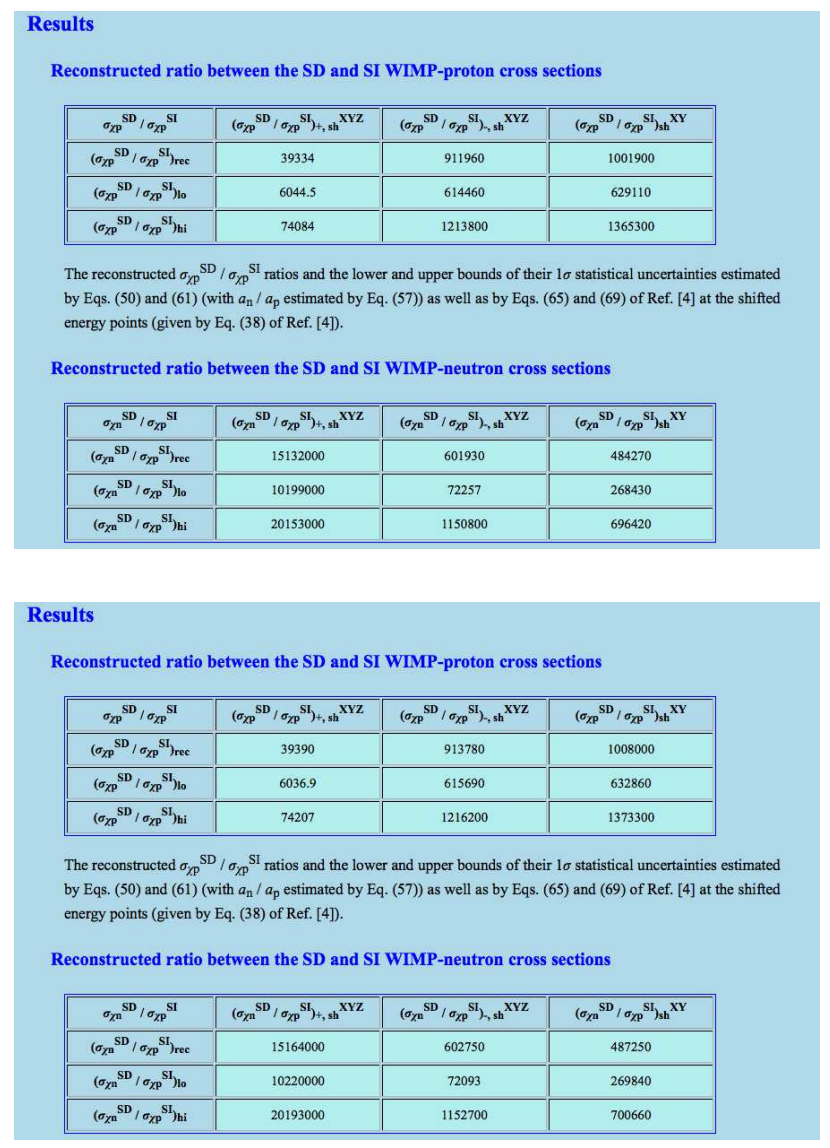

Fig. 4. The reconstructed ratios between the SD and SI WIMP-nucleon couplings, $\sigma_{\chi(\mathrm{p}, \mathrm{n})}^{\mathrm{SD}} / \sigma_{\chi \mathrm{p}}^{\mathrm{SI}}$. As usual, the elastic nuclear form factors given in Eqs. (2.1) and (2.2) have been used for determining $\sigma_{\chi(\mathrm{p}, \mathrm{n})}^{\mathrm{SD}} / \sigma_{\chi \mathrm{p}}^{\mathrm{SI}}$ in the upper and lower frames, respectively.

assumption $^{5}$, one could still use the mean value and the overlap of these two results to roughly (and somehow naively) give a $1 \sigma$ range of

$$
\frac{a_{\mathrm{n}}}{a_{\mathrm{p}}} \simeq 0.89_{-0.30}^{+0.26}
$$

\subsection{Ratios of the SD and SI WIMP-nucleon couplings $\sigma_{\chi(\mathrm{p}, \mathrm{n})}^{\mathrm{SD}} / \sigma_{\chi \mathrm{p}}^{\mathrm{SI}}$}

In Figs. $4 \mathrm{I}$ show the reconstructed $\sigma_{\chi(\mathrm{p}, \mathrm{n})}^{\mathrm{SD}} / \sigma_{\chi \mathrm{p}}^{\mathrm{SI}}$ ratios and the lower and upper bounds of their $1 \sigma$ statistical uncertainties estimated by Eqs. (3.9), (3.10) and

\footnotetext{
${ }^{5}$ See also discussions in Sec. 2.4.
} 
(3.21) of Shan (2011) (with $a_{\mathrm{n}} / a_{\mathrm{p}}$ estimated by Eq. (3.16) of Shan (2011)) as well as by Eqs. (3.25) and (3.29) of Shan (2011) at the shifted energy points.

By using the data sets of ${ }^{19} \mathrm{~F},{ }^{127} \mathrm{I}$ and ${ }^{28} \mathrm{Si}$ targets (labeled with the superscript "XYZ") or combining that of ${ }^{23} \mathrm{Na}$ or ${ }^{131} \mathrm{Xe}$ with the (common) data set of ${ }^{76} \mathrm{Ge}$ (labeled with the superscript "XY"), one can use the mean value and the overlap of these two results to roughly (and somehow naively) give a $1 \sigma$ range of

$$
\frac{\sigma_{\chi \mathrm{p}}^{\mathrm{SD}}}{\sigma_{\chi \mathrm{p}}^{\mathrm{SI}}} \simeq 9.61_{-3.28}^{+2.55} \times 10^{5}, \quad \frac{\sigma_{\chi \mathrm{n}}^{\mathrm{SD}}}{\sigma_{\chi \mathrm{p}}^{\mathrm{SI}}} \simeq 5.45_{-2.75}^{+1.56} \times 10^{5} .
$$

Then, firstly, from these results one can further obtain that ${ }^{6,7}$

$$
\left|\frac{a_{\mathrm{n}}}{a_{\mathrm{p}}}\right| \simeq 0.75_{-0.23}^{+0.15} \text {. }
$$

Secondly, combining the results in Eq. (2.13) with $\sigma_{\chi \mathrm{p}}^{\mathrm{SI}}$ given in Eq. (2.11), one can also obtain that ${ }^{8}$

$$
\sigma_{\chi \mathrm{p}}^{\mathrm{SD}} \simeq 4.14_{-1.56}^{+1.47} \times 10^{-3} \mathrm{pb}, \quad \sigma_{\chi \mathrm{n}}^{\mathrm{SD}} \simeq 2.35_{-1.31}^{+0.87} \times 10^{-3} \mathrm{pb} .
$$

These results give in turn that ${ }^{9}$

$$
\left|a_{\mathrm{p}}\right| \simeq 0.108_{-0.020}^{+0.019}, \quad\left|a_{\mathrm{n}}\right| \simeq 0.081_{-0.023}^{+0.015} .
$$

$$
\begin{aligned}
& { }^{6} \text { Here I have used } \\
& \qquad \begin{array}{r}
\sigma\left(\left|\frac{a_{\mathrm{n}}}{a_{\mathrm{p}}}\right|\right)=\frac{1}{2}\left|\frac{a_{\mathrm{n}}}{a_{\mathrm{p}}}\right|\left[\sigma^{2}\left(\sigma_{\chi \mathrm{n}}^{\mathrm{SD}} / \sigma_{\chi \mathrm{p}}^{\mathrm{SI}}\right) /\left(\sigma_{\chi \mathrm{n}}^{\mathrm{SD}} / \sigma_{\chi \mathrm{p}}^{\mathrm{SI}}\right)^{2}\right. \\
\left.+\sigma^{2}\left(\sigma_{\chi \mathrm{p}}^{\mathrm{SD}} / \sigma_{\chi \mathrm{p}}^{\mathrm{SI}}\right) /\left(\sigma_{\chi \mathrm{p}}^{\mathrm{SD}} / \sigma_{\chi \mathrm{p}}^{\mathrm{SI}}\right)^{2}\right]^{1 / 2},
\end{array}
\end{aligned}
$$

and neglected the correlation term in the bracket:

$$
-2 \operatorname{cov}\left(\sigma_{\chi \mathrm{n}}^{\mathrm{SD}} / \sigma_{\chi \mathrm{p}}^{\mathrm{SI}}, \sigma_{\chi \mathrm{p}}^{\mathrm{SD}} / \sigma_{\chi \mathrm{p}}^{\mathrm{SI}}\right) /\left(\sigma_{\chi \mathrm{n}}^{\mathrm{SD}} / \sigma_{\chi \mathrm{p}}^{\mathrm{SI}}\right)\left(\sigma_{\chi \mathrm{p}}^{\mathrm{SD}} / \sigma_{\chi \mathrm{p}}^{\mathrm{SI}}\right),
$$

since the $1 \sigma$ uncertainties given in Eq. (2.13) are not the exact but only rough estimates from the overlaps of two results given in Figs. 4.

${ }^{7}$ Remind that the results given in the second and third columns of the tables in Figs. 4 are reconstructed with the $a_{\mathrm{n}} / a_{\mathrm{p}}$ ratio given in the last columns of the tables in Figs. 3.

${ }^{8}$ Here I have used

$$
\sigma\left(\sigma_{\chi(\mathrm{p}, \mathrm{n})}^{\mathrm{SD}}\right)=\left[\left(\sigma_{\chi \mathrm{p}}^{\mathrm{SI}}\right)^{2} \sigma^{2}\left(\sigma_{\chi(\mathrm{p}, \mathrm{n})}^{\mathrm{SD}} / \sigma_{\chi \mathrm{p}}^{\mathrm{SI}}\right)+\left(\sigma_{\chi(\mathrm{p}, \mathrm{n})}^{\mathrm{SD}} / \sigma_{\chi \mathrm{p}}^{\mathrm{SI}}\right)^{2} \sigma^{2}\left(\sigma_{\chi \mathrm{p}}^{\mathrm{SI}}\right)\right]^{1 / 2}
$$

and neglected the correlation term in the bracket:

$$
2 \operatorname{cov}\left(\sigma_{\chi \mathrm{p}}^{\mathrm{SI}}, \sigma_{\chi(\mathrm{p}, \mathrm{n})}^{\mathrm{SD}} / \sigma_{\chi \mathrm{p}}^{\mathrm{SI}}\right) /\left(\sigma_{\chi \mathrm{p}}^{\mathrm{SI}}\right)\left(\sigma_{\chi(\mathrm{p}, \mathrm{n})}^{\mathrm{SD}} / \sigma_{\chi \mathrm{p}}^{\mathrm{SI}}\right)
$$

by assuming that two independent data sets with the ${ }^{76} \mathrm{Ge}$ target and other two independent data sets with the ${ }^{28} \mathrm{Si}$ target have been used for determining $\sigma_{\chi \mathrm{p}}^{\mathrm{SI}}$ and $\sigma_{\chi(\mathrm{p}, \mathrm{n})}^{\mathrm{SD}} / \sigma_{\chi \mathrm{p}}^{\mathrm{SI}}$.

${ }^{9}$ Since

$$
\sigma_{\chi(\mathrm{p}, \mathrm{n})}^{\mathrm{SD}}=\left(\frac{24}{\pi}\right) G_{F}^{2} m_{\mathrm{r},(\mathrm{p}, \mathrm{n})}^{2}\left|a_{(\mathrm{p}, \mathrm{n})}\right|^{2}
$$


On the other hand, one can also use the reconstructed $a_{\mathrm{n}} / a_{\mathrm{p}}$ ratio given in Eq. (2.12) and one of the two results given in Eq. (2.19) to obtain that ${ }^{10}$

$$
\sigma_{\chi \mathrm{p}}^{\mathrm{SD}} \simeq 2.97_{-2.60}^{+2.05} \times 10^{-3} \mathrm{pb}, \quad \sigma_{\chi \mathrm{n}}^{\mathrm{SD}} \simeq 3.28_{-2.53}^{+2.24} \times 10^{-3} \mathrm{pb} .
$$

These results can also give that

$$
\left|a_{\mathrm{p}}\right| \simeq 0.091_{-0.040}^{+0.032}, \quad\left|a_{\mathrm{n}}\right| \simeq 0.096_{-0.037}^{+0.033} .
$$

It can be found that, not surprisingly, the statistical uncertainties on the reconstructed $\sigma_{\chi(\mathrm{p}, \mathrm{n})}^{\mathrm{SD}}$ given in Eq. (2.26) are $\sim 2$ or 3 times larger than those given in Eq. (2.19): Since $\sigma_{\chi(\mathrm{p}, \mathrm{n})}^{\mathrm{SD}} / \sigma_{\chi \mathrm{p}}^{\mathrm{SI}}$ reconstructed with the $\mathrm{F}+\mathrm{I}+\mathrm{Si}$ combination involve already the reconstructed $a_{\mathrm{n}} / a_{\mathrm{p}}$ ratio given in Eq. (2.12), the uncertainties on $\sigma_{\chi(\mathrm{p}, \mathrm{n})}^{\mathrm{SD}}$ given in Eq. (2.26) are thus overestimated. Secondly, although the reconstructed $\sigma_{\chi \mathrm{p}}^{\mathrm{SD}}$ and $\sigma_{\chi \mathrm{n}}^{\mathrm{SD}}$ given in Eqs. (2.19) and (2.26) have overlaps, these results seem not to match to each other very well; $\sigma_{\chi \mathrm{n}}^{\mathrm{SD}}$ given in Eq. (2.26) is even larger than $\sigma_{\chi \mathrm{p}}^{\mathrm{SD}}$ there although the $a_{\mathrm{n}} / a_{\mathrm{p}}$ ratios given in Eqs. (2.12) and (2.16) are $<1$. One possible explanation is that the $a_{\mathrm{n}} / a_{\mathrm{p}}$ ratio given in Eq. (2.12) would be overestimated. This can be seen by comparing the $a_{\mathrm{n}} / a_{\mathrm{p}}$ ratio given in Eq. (2.12) to that given in Eq. (2.16) estimated (somehow independently) by the results given in Eq. (2.13).

Nevertheless, the analyses given here show that, firstly, once one can estimate the SI WIMP-nucleon coupling/cross section, $\left|f_{\mathrm{p}}\right|$ or $\sigma_{\chi \mathrm{p}}^{\mathrm{SI}}$, and (one of) the ratios between the SD and SI WIMP-nucleon cross sections, and/or the ratio between two SD WIMP-nucleon couplings, the other couplings/cross sections could in principle be estimated. Secondly, although the method under the assumption of the

and $m_{\chi} \sim 120 \mathrm{GeV}$, one has

$$
\left|a_{(\mathrm{p}, \mathrm{n})}\right|=\sqrt{\frac{\pi}{24}} \frac{\sqrt{\sigma_{\chi(\mathrm{p}, \mathrm{n})}^{\mathrm{SD}}}}{G_{F} m_{\mathrm{r},(\mathrm{p}, \mathrm{n})}} \approx \sqrt{\frac{\pi}{24}} \frac{\sqrt{\sigma_{\chi(\mathrm{p}, \mathrm{n})}^{\mathrm{SD}}}}{G_{F} m_{(\mathrm{p}, \mathrm{n})}}
$$

and

$$
\sigma\left(\left|a_{(\mathrm{p}, \mathrm{n})}\right|\right)=\sqrt{\frac{\pi}{96}} \frac{\sigma\left(\sigma_{\chi(\mathrm{p}, \mathrm{n})}^{\mathrm{SD}}\right)}{G_{F} m_{\mathrm{r},(\mathrm{p}, \mathrm{n})} \sqrt{\sigma_{\chi(\mathrm{p}, \mathrm{n})}^{\mathrm{SD}}}} \approx \sqrt{\frac{\pi}{96}} \frac{\sigma\left(\sigma_{\chi(\mathrm{p}, \mathrm{n})}^{\mathrm{SD}}\right)}{G_{F} m_{(\mathrm{p}, \mathrm{n})} \sqrt{\sigma_{\chi(\mathrm{p}, \mathrm{n})}^{\mathrm{SD}}}} .
$$

${ }^{10}$ Here I have used

$$
\sigma\left(\sigma_{\chi(\mathrm{p}, \mathrm{n})}^{\mathrm{SD}}\right)=\sigma_{\chi(\mathrm{p}, \mathrm{n})}^{\mathrm{SD}}\left[\sigma^{2}\left(\sigma_{\chi(\mathrm{n}, \mathrm{p})}^{\mathrm{SD}}\right) /\left(\sigma_{\chi(\mathrm{n}, \mathrm{p})}^{\mathrm{SD}}\right)^{2}+4 \sigma^{2}\left(a_{\mathrm{n}} / a_{\mathrm{p}}\right) /\left(a_{\mathrm{n}} / a_{\mathrm{p}}\right)^{2}\right]^{1 / 2},
$$

and neglected the correlation term in the bracket:

$$
\mp 4 \operatorname{cov}\left(\sigma_{\chi(\mathrm{n}, \mathrm{p})}^{\mathrm{SD}}, a_{\mathrm{n}} / a_{\mathrm{p}}\right) /\left(\sigma_{\chi(\mathrm{n}, \mathrm{p})}^{\mathrm{SD}}\right)\left(a_{\mathrm{n}} / a_{\mathrm{p}}\right)
$$

since the $1 \sigma$ uncertainties given in Eq. (2.12) as well as in Eq. (2.19) are not the exact but only rough estimates from the overlaps of the results given in Figs. 2, 3 and 4. The "- $(+)$ " sign in Eq. (2.25) is for the case with protons (neutrons). 
SD dominant WIMP interaction would overestimate (or underestimate, depending on the combination of the used targets (Shan 2011)) the $a_{\mathrm{n}} / a_{\mathrm{p}}$ ratio, the reconstructed result(s) could still be useful for at least determining the correct sign of $a_{\mathrm{n}} / a_{\mathrm{p}}$. Moreover, the WIMP couplings/cross sections estimated in different way would be self-cross-checks to each other and the (in)compatibility between the reconstructed results would also help us to check the usefulness of the analyzed data sets offered from different experiments with different detector materials.

\section{Input setup for generating pseudodata}

In Table 1 I give finally the input setup for generating the pseudodata sets used in the analyses demonstrated in the previous section. For comparison, the reconstructed results shown in the previous section are also summarized here.

It can be found that, firstly, not only the WIMP mass given in Eq. (2.4) and the result reconstructed with the input nuclear form factor (lower frame of Figs. 1), but even the mass reconstructed with the "wrong" form factor (upper frame) can match the input WIMP mass very well: the deviations between the input and the reconstructed values are only $\sim 13 \%$ (with the wrong nuclear form factor) or even only $\sim 6 \%$ (with the input one). As discussed earlier, this indicates that, for the first approximation of giving/constraining the most plausible range of the WIMP mass, the uncertainty on the nuclear form factor could be safely neglected.

Secondly, all WIMP-nucleon couplings/cross sections as well as the ratios between them have also been reconstructed with only $\sim 5 \%$ to $\lesssim 40 \%$ deviations from the input/theoretically estimated values. Although the SI WIMP coupling $\left|f_{\mathrm{p}}\right|$ estimated with the input (larger) local Dark Matter density (lower frame of Figs. 2) is underestimated (Shan 2011), one can at least give an upper bound on $\left|f_{\mathrm{p}}\right|$. Meanwhile, although the $a_{\mathrm{n}} / a_{\mathrm{p}}$ ratio given in Eq. (2.12) is overestimated, in Sec. 2.4 we have demonstrated that by combining different methods for estimating different (ratios between the) WIMP couplings/cross sections, one could in principle observe/confirm the (in)compatibility between these results and probably correct the reconstructed values.

Moreover, for generating pseudodata, we have used the shifted Maxwellian velocity distribution:

$$
f_{1, \mathrm{sh}}(v)=\frac{1}{\sqrt{\pi}}\left(\frac{v}{v_{\mathrm{e}} v_{0}}\right)\left[e^{-\left(v-v_{\mathrm{e}}\right)^{2} / v_{0}^{2}}-e^{-\left(v+v_{\mathrm{e}}\right)^{2} / v_{0}^{2}}\right],
$$

with the Sun's Galactic orbital velocity $v_{0}=230 \mathrm{~km} / \mathrm{s} ; v_{\mathrm{e}}$ is the time-dependent Earth's velocity in the Galactic frame:

$$
v_{\mathrm{e}}(t)=v_{0}\left[1.05+0.07 \cos \left(\frac{2 \pi\left(t-t_{\mathrm{p}}\right)}{1 \mathrm{yr}}\right)\right],
$$

the date on which the Earth's velocity relative to the WIMP halo is maximal has been set as $t_{\mathrm{p}}=140 \mathrm{~d}$. Although these values for the astronomical setup are non-standard, we would like to stress that, firstly, for using the AMIDAS package 


\begin{tabular}{||c|c|c|l||}
\hline \hline Property & Reconstructed value & Input/Estimated value & Remarks \\
\hline \hline$m_{\chi}$ & $120_{-40}^{+70} \mathrm{GeV}$ & $130 \mathrm{GeV}$ & \\
\hline \hline$\sigma_{\chi \mathrm{p}}^{\mathrm{SI}}$ & $4.31_{-0.69}^{+1.01} \times 10^{-9} \mathrm{pb}$ & $4 \times 10^{-9} \mathrm{pb}$ & $f_{\mathrm{n}}=f_{\mathrm{p}}$ \\
\hline$\left|f_{\mathrm{p}}\right|^{2}$ & $9.00_{-1.44}^{+2.10} \times 10^{-18} \mathrm{GeV}^{-4}$ & $9.305 \times 10^{-18} \mathrm{GeV}^{-4}$ & $\dagger$ \\
\hline$\left|f_{\mathrm{p}}\right|$ & $3.00_{-0.24}^{+0.35} \times 10^{-9} \mathrm{GeV}^{-2}$ & $3.050 \times 10^{-9} \mathrm{GeV}^{-2}$ & $\dagger$ \\
\hline \hline$a_{\mathrm{p}}$ & $0.108_{-0.020}^{+0.019}$ & 0.1 & \\
$a_{\mathrm{n}}$ & $0.081_{-0.023}^{+0.015}$ & 0.07 & \\
\hline$a_{\mathrm{n}} / a_{\mathrm{p}}$ & $0.89_{-0.30}^{+0.26}, 0.75_{-0.23}^{+0.15}$ & 0.7 & \\
\hline$\sigma_{\chi \mathrm{p}}^{\mathrm{SD}}$ & $4.14_{-1.56}^{+1.47} \times 10^{-3} \mathrm{pb}$ & $3.51 \times 10^{-3} \mathrm{pb}$ & $\dagger$ \\
$\sigma_{\chi \mathrm{n}}^{\mathrm{SD}}$ & $2.35_{-1.31}^{+0.87} \times 10^{-3} \mathrm{pb}$ & $1.72 \times 10^{-3} \mathrm{pb}$ & $\dagger$ \\
\hline$\sigma_{\chi \mathrm{p}}^{\mathrm{SD}} / \sigma_{\chi \mathrm{p}}^{\mathrm{SI}}$ & $9.61_{-3.28}^{+2.55} \times 10^{5}$ & $8.77 \times 10^{5}$ & $\dagger$ \\
$\sigma_{\chi \mathrm{n}}^{\mathrm{SD}} / \sigma_{\chi \mathrm{p}}^{\mathrm{SI}}$ & $5.45_{-2.75}^{+1.56} \times 10^{5}$ & $4.30 \times 10^{5}$ & $\dagger$ \\
\hline \hline$F_{\mathrm{SI}}^{2}(Q)$ & & $F_{\mathrm{SI}}^{2}(Q) \mathrm{in} \mathrm{Eq.}(2.2)$ & \\
$F_{\mathrm{SD}}^{2}(Q)$ & & $F_{\mathrm{TS}}(Q) \mathrm{in} \mathrm{Eq.}(2.3)$ & \\
\hline \hline$\rho_{0}$ & & $0.4 \mathrm{GeV} / \mathrm{cm}{ }^{3}$ & \\
\hline$t_{\mathrm{p}}$ & & $300 \mathrm{~d}$ & \\
$t_{\text {expt }}$ & & $230 \mathrm{~km} / \mathrm{s}$ & \\
\hline$v_{0}$ & & $226.6 \mathrm{~km} / \mathrm{s}$ & \\
$v_{\max }$ & & & \\
$v_{\mathrm{e}}\left(t_{\text {expt }}\right)$ & & & $140 \mathrm{~d}$ \\
\hline \hline
\end{tabular}

Table 1. The input setup for generating the pseudodata sets used in the analyses demonstrated in this article. The theoretically estimated values and the reconstructed results shown in the previous section are also given. $\dagger$ : estimated for $130 \mathrm{GeV} m_{\chi}$.

and website to analyze (real) data sets, one needs only the form factors for SI and/or SD WIMP-nucleaus cross sections, prior knowledge/assumptions about the WIMP velocity distribution $f_{1}(v)$ and local density $\rho_{0}$ (except the estimation of the SI WIMP-nucleon coupling $\left|f_{\mathrm{p}}\right|^{2}$ ) are not required. Secondly, as shown in the previous section, such non-standard values would not affect the reconstructed results.

\section{Summary}

In this article I demonstrated the data analysis procedures for extrating WIMP properties by using theoretically generated pseudodata for different target nu- 
clei. As an extension as well as the complementarity of our earlier theoretical works, I combined reconstructed results of the (ratios between different) WIMP couplings/cross sections on nucleons to estimate each individual coupling/cross section. Hopefully, the AMIDAS package and website as well as this demonstration can help our experimental colleagues to analyze their real direct detection data in the near future and to determine (at least rough ranges of) properties of halo Dark Matter particles.

\section{Acknowledgments}

The author appreciates the ILIAS Project and the Physikalisches Institut der Universität Tübingen for kindly providing the opportunity of the collaboration and the technical support of the AMIDAS website. The author would also like to thank the friendly hospitality of the National Institute for Nuclear and High Energy Physics (NIKHEF) where part of this work was completed. This work was partially supported by the National Science Council of R.O.C. under contract no. NSC-992811-M-006-031 as well as by the National Center of Theoretical Sciences (South), R.O.C..

\section{References}

AMIDAS website, see http://pisrv0.pit.physik.uni-tuebingen.de/darkmatter/amidas/.

Catena, R. \& Ullio, P. 2010, JCAP 1008, 004.

DAMNED online tool, see http://pisrv0.pit.physik. uni-tuebingen.de/darkmatter/index1.html.

Drees, M. \& Shan, C.-L. 2007, JCAP 0706, 011.

Drees, M. \& Shan, C.-L. 2008, JCAP 0806, 012.

ILIAS Project, see http://www-ilias.cea.fr/.

Pato, M., Agertz, O., Bertone, G., Moore, B. \& Teyssier, R. 2010, PRD 82, 023531.

Salucci, P., Nesti, F., Gentile G. \& Martins, C. F. 2010, A $6 A$ 523, A83.

Shan, C.-L. 2009, arXiv:0910.1971 [astro-ph.IM].

Shan, C.-L. 2010, AIP Conf. Proc. 1200, 1031.

Shan, C.-L. 2011, JCAP 1107, 005.

Shan, C.-L. 2011, arXiv:1103.0481 [hep-ph]. 